\title{
Argon indirect ophthalmoscopic photocoagulation: reduced potential phototoxicity with a fixed safety filter
}

\author{
Marc M Whitacre, Nubar Manoukian, Martin A Mainster
}

Ophthalmology, Kansas University Medical Center, Kansas City, Kansas, USA M M Whitacre M A Mainster

\section{Coherent Medical} Division, Palo Alto, California, USA N Manoukian

Correspondence to: Marc $M$ Whitacre MD, Department of Ophthalmology, Kansas University Medical Center, USA.

Accepted for publication 16 November 1989
Department of 39th and Rainbow Boulevard, Kansas City, Kansas, 66103,

\begin{abstract}
A new argon indirect ophthalmoscopic photocoagulator is presented which uses a red helium-neon laser aiming beam. An interference filter protects the operator from green or blue-green treatment beam reflections without impairing visualisation of the aiming beam or significant retinal anatomy. The protective filter is fixed in place, eliminating the weight, noise, and potential failure of mechanically switched filters. The red aiming beam has a negligible potential for producing photochemical retinal damage in the patient or operator.
\end{abstract}

Binocular indirect ophthalmoscopic laser photocoagulation is useful for treating a variety of retinal disorders in the consulting room or operating room. ${ }^{1}$ Previously described laser indirect ophthalmoscopes (LIOs) have used the same wavelength for aiming and treatment beams and either a switched or fixed protective filter system.

Switched protective filters remain out of the operator's view during fundus and aiming beam observation. ${ }^{12}$ When the foot switch is

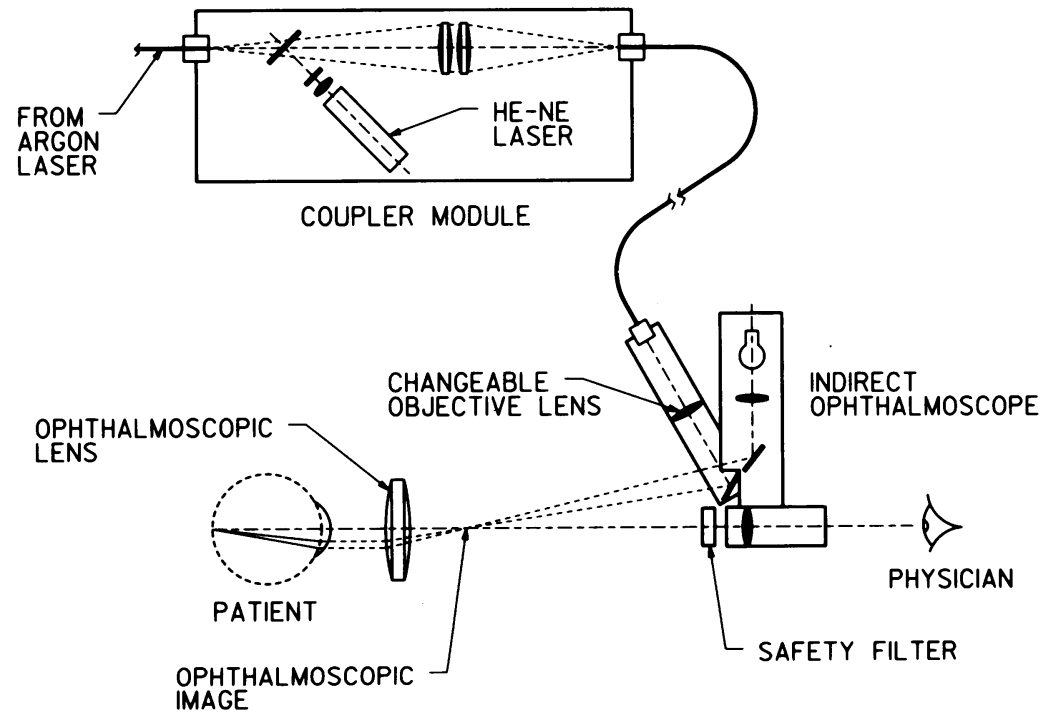

Figure 1: The dual wavelength indirect ophthalmoscope photocoagulator consists of an argon laser console, a coupler module, and an indirect ophthalmoscope. A primary fibreoptic cable attaches the argon laser console to the coupler module, and a secondary fibreoptic cable attaches the module to the ophthalmoscope. The coupler module contains the ophthalmoscope's power supply and a $1 \mathrm{~mW}$ red $\mathrm{He}$-Ne laser. The module combines the aiming and treatment beams into a single coaxial beam that is focused into the secondary fibreoptic cable. The coaxial beam exits the secondary fibre at the ophthalmoscope and is focused by a changeable objective lens before it reflects off a mirror. The operator directs the reflected beam into the patient's eye with a standard aspheric ophthalmoscopic lens. The user is protected from argon laser treatment beam reflections by an interference safety filter that blocks light between $455 \mathrm{~nm}$ and $525 \mathrm{~nm}$ but transmits light at shorter and longer wavelengths to permit effective ophthalmoscopy and visualisation of the red aiming beam. depressed, they move into place, blocking treatment beam reflections. Switched filter systems provide unimpeded fundus observation, but they are heavy, noisy, and subject to mechanical failure, and the operator has no protection when viewing the aiming beam.

Fixed protective filter systems remain in place during observation and treatment. ${ }^{34}$ Since they have no moving parts, fixed filters are silent and intrinsically reliable. When aiming and treatment beams are of the same wavelength, however, a fixed filter may reduce the intensity of the observable aiming beam to an unsatisfactory level. Increasing the intensity of the aiming beam is a poor solution to this problem because it increases the patient's risk of iatrogenic thermal or photochemical retinal damage.

Since there are problems with both switched and fixed protective filters when a single wavelength is used for LIO aiming and treatment beams, we have helped to develop a dual wavelength LIO with a red $(632.8 \mathrm{~nm}) \mathrm{He}-\mathrm{Ne}$ laser aiming beam and a green $(514.5 \mathrm{~nm})$ or blue green $(488 \mathrm{~nm}+514 \cdot 5 \mathrm{~nm})$ argon laser treatment beam.

\section{Material and methods}

An argon laser console (Coherent Medical Division, Model 920/930) is attached by a primary fibreoptic cable to a coupler module. The module is attached by a secondary fibreoptic cable to an indirect ophthalmoscope (Keeler Instruments, Fison model with modifications). The module contains the ophthalmoscope's power supply and a $1 \mathrm{~mW}$ red $\mathrm{He}-\mathrm{Ne}$ laser. The optical system is illustrated in Figure 1.

The coupler module combines aiming and treatment beams into a single coaxial beam that is focused into the secondary fibreoptic cable. The coaxial beam leaves the secondary fibre at the ophthalmoscope and is focused by a changeable objective lens before it reflects off a mirror. The operator directs the reflected beam into the patient's eye using a conventional aspheric ophthalmoscopic lens.

The user is protected from argon laser treatment beam reflections by an interference safety filter that blocks light between $455 \mathrm{~nm}$ and $525 \mathrm{~nm}$. The safety filter transmits light at shorter and longer wavelengths to permit effec ive ophthalmoscopy and visualisation of the red aiming beam. The He-Ne laser aiming beam is adjustable over a wide range of intensities for different viewing circumstances.

The aerial retinal image of an indirect ophthalmoscope is located anterior to the hand-held ophthalmoscopic lens, at a working distance 
from the operator determined by the power of the lens and the patient's eye. When the laser beam is focused precisely at that distance, retinal spot size is smallest and retinal irradiance is highest. The dual wavelength LIO produces a minimum retinal spot size of $320 \mu \mathrm{m}$ with a 20dioptre ophthalmoscopic lens. Since deviations as little as $50 \mathrm{~mm}$ from the focal plane of the ophthalmoscopic lens can double the spot size and reduce retinal irradiance by a factor of 4 , changeable objective lenses $(320 \mathrm{~mm}, 420 \mathrm{~mm}$, and $520 \mathrm{~mm}$ focal lengths) are used to focus the laser beam at the proper working distance for different users. Deliberate deviation from the focal plane is a useful method of increasing retinal spot size.

\section{Results}

The dual wavelength LIO has the same uses as those reported for single wavelength LIOs. ${ }^{134}$ In the consulting room the LIO has been useful for panretinal photocoagulation through vitreous haemorrhage, for treating peripheral retinal breaks, for photocoagulation following vitreoretinal surgery requiring intraocular gas, and for treating patients whose frailty or obesity precludes conventional biomicroscopic photocoagulation. Additional operating room applications include photocoagulation in children under general anaesthesia and retinopexy after subretinal fluid drainage. With its built-in argon filters, the LIO can also be used to direct retinal endophotocoagulation when corneal or lenticular opacities preclude safe treatment with an argon laser beam originating outside the eye. ${ }^{5}$

The fixed safety filter gives the fundus a slightly yellowish tint but does not interfere with the detection of fundus details such as small retinal holes. The LIO is not useful for procedures such as macular photocoagulation that require precision in burn placement, because image magnification is too low and the patient's or operator's head may move. If motion is a problem in other procedures, decreasing the duration of exposure and increasing laser power can help limit size of burn, as can the use of repetitive mode photocoagulation, which provides a series of burns with a single depression of the foot switch.

\section{Discussion}

Clinical infrared laser systems generally use a visible red $\mathrm{He}-\mathrm{Ne}$ laser aiming beam. ${ }^{67}$ Since infrared reflections can be blocked by a fixed safety filter that does not impair visualisation of the aiming beam or operating field, infrared laser safety systems are usually silent, lightweight, and intrinsically reliable. The safety system of the dual wavelength argon LIO has these same advantages. A similar system could be used with conventional biomicroscopic argon photocoagulators.

The dual wavelength LIOs red $\mathrm{He}-\mathrm{Ne}$ laser aiming beam has the theoretical advantage of lower light scattering and better penetration of hazy ocular media than argon laser green or bluegreen aiming beams. ${ }^{8}$ It has another possible advantage unrelated to clinical applications. Experimental studies have shown that susceptibility to photic retinopathy increases with decreasing wavelength: ultraviolet and blue light have the greatest potential phototoxicity, while red light has negligible damage potential. ${ }^{9-11}$ Studies have also shown that phototoxicity may be cumulative, so that light exposure increases the risk of damage to subsequent light exposure. ${ }^{12}$

In a conventional switched-filter LIO the user is unprotected when viewing argon blue-green or green aiming beam reflections. Furthermore, intense aiming beams are often needed for treating eyes with hazy ocular media. A recent study has demonstrated a subtle blue yellow colour contrast defect in long-term argon photocoagulator operators. ${ }^{13}$ This defect is demonstrable only with extremely sensitive computerised testing, and its clinical significance is uncertain. If the defect is significant, however, the red aiming beam of the dual wavelength LIO system has an additional theoretical advantage: negligible potential retinal phototoxicity for the patient and operator. ${ }^{811}$

This research was supported in part by the Kansas Lions Sight Foundation, Inc, and Research to Prevent Blindness, Inc. M M Whitacre and M A Mainster do not have a proprietary interest in any indirect ophthalmoscope, laser system, or manufacturer. N Manoukian is an employee of Coherent Medical Division which produces ophthalmic laser systems.

1 Friberg TR. Clinical experience with a binocular indirect ophthalmoscope laser delivery system. Retina 1987; 7: 28-31.

2 Grisolano J, Peyman GA. An automatic laser filter for the indirect ophthalmoscope. Retina 1987; 7: 32-3.

3 Mizuno K. Binocular indirect argon laser photocoagulator. $\mathrm{Br}$ f Ophthalmol 1981; 65: 425-8.

4 Mizuno K, Takaku Y. Dual delivery system for argon laser photocoagulation: improved techniques of the binocular indirect argon laser photocoagulator. Arch Ophthalmol 1983; 101: 648-52.

5 Hampton GR. Argon endophotocoagulation with indirect ophthalmoscopy. Arch Ophthalmol 1987; 105: 132

6 Mainster MA, Sliney DH, Belcher CD, III, Buzney SM. Laser photodisruptors: damage mechanisms, instrument design
pher and safety. Ophthalmology 1983; 90: 973-91.

7 L'Esperance FA Jr. Ophthalmic lasers: photocoagulation, photoradiation and surgery. 3rd ed. St. Louis: Mosby, 1989: 2: 751-80.

8 Mainster MA. Wavelength selection in macular photocoagulation: tissue optics, thermal effects and laser systems. Ophthalmology 1986; 93: 952-8.

9 Ham WT Jr, Mueller HA, Sliney DH. Retinal sensitivity to damage from short wavelength light. Nature 1976; 260: 153-5.

10 Mainster MA, Ham WT Jr, Delori FC. Potential retinal hazards: instrument and environmental light sources. Ophthalmology 1983; 90: 927-32.

11 Mainster MA. Photic retinal injury. In: Ryan SJ, ed. Retina. St Louis: Mosby, 1989: 2: 749-57.

12 Greiss GA, Blankenstein MF. Additivity and repair of actinic retinal lesions. Invest Ophthalmol Vis Sci 1981; 20: 803-7.

13 Gunduz K, Arden GB. Changes in colour contrast sensitivity associated with operating argon lasers. $\mathrm{Br} \mathcal{F}$ Ophthalmol 1989; 73: 241-6. 